\title{
Paper technology
}

\section{Javier Brugés Martelo*, Mattias Andersson, Consolatina Liguori and Jan Lundgren Three-dimensional scanning electron microscopy used as a profilometer for the surface characterization of polyethylene-coated paperboard}

https://doi.org/10.1515/npprj-2021-0003

Received January 8, 2021; accepted February 6, 2021; previously published online March 2, 2021

Abstract: In food packaging, low-density polyethylene (PE) coating is applied to paperboards to act as a functional barrier and to provide the smoothness required to enhance printability. These characteristics are related to the material's surface roughness, the parameter monitored during the manufacturing process. Measurement of surface roughness using optical profilometry has gained importance in the paper industry. The optical instruments used to measure surface roughness are limited spatially by the relationship with the light wavelength at which they operate. A scanning electron microscope (SEM) is an alternative for overcoming the spatial resolution limitation, and the use of stereo-photogrammetry on SEM images can be seen as an alternative profilometry technique to measure surface roughness. In this investigation, the surface topography of industrially manufactured highquality PE-coated paperboard was studied, comparing the SEM stereo-photogrammetry technique with a reference profilometry method, i. e., chromatic confocal microscopy (CCM). We found close agreement between the calculated surface roughness and the results of the techniques used and compared them according to the new ISO $25178 \mathrm{Ge}$ ometric Product Specifications. We concluded that SEM stereo-photogrammetry provides comparable accurate alternative profilometry method for characterizing the surface roughness of $\mathrm{PE}$-coated paperboard in the micrometer scale.

\footnotetext{
*Corresponding author: Javier Brugés Martelo, Electronic Design Department, Mid Sweden University, Holmagatan 10, Sundsvall, Sweden, e-mail: javier.bruges@miun.se, ORCID: https://orcid.org/0000-0002-7240-3840

Mattias Andersson, Department of Design, Mid Sweden University, Örnsköldsvik, Sweden, e-mail: mattias.andersson@miun.se Consolatina Liguori, Department of Industrial Engineering (DIIn), University of Salerno, Fisciano, SA, Italy, e-mail: tliguori@unisa.it Jan Lundgren, Electronic Design Department, Mid Sweden University, Holmagatan 10, Sundsvall, Sweden, e-mail: jan.lundgren@miun.se
}

Keywords: inter-instrument comparison; ISO 25178; material characterization; optical profilometry; surface roughness.

\section{Introduction}

Paperboard grades are classified depending on their functionality, with food packaging paperboard being higher in optical and surface quality. Different surface finishes and coatings are specified to produce the desired product functionalities and qualities. In the food packaging industry, polyethylene (PE) coating is applied to paperboards because of its excellent barrier functionality against moisture and the resulting smooth final surface texture, both of which are important for visual appeal and the intended product quality. Surface roughness is measured on the paperboard through its correlation with optical properties, such as gloss uniformity, which are related to the final product specifications (Béland and Bennett 2000).

\section{Industrial surface characterization of paperboard}

In PE coating of paperboard, the measurement of surface roughness is used to monitor the smoothness of the final product, which is a surface parameter that affects printability. The standard for measuring surface roughness in the paper and board industry is based on the air leak method, i.e. Bendtsen, Bekk and Parker print surf (PPS) (Enomae and Onabe 1997). The measurement instrument estimates surface roughness by measuring the rate at which air escapes from a system consisting of metal plates sandwiched between paperboard samples, and the results are correlated with the roughness parameter of the sample. The calibration used to estimate roughness values is limited by the range of spatial distribution of the surface of the product grade. Although what constitutes a good agreement between paper and paperboard grades and their surface parameters is well defined, the associated measurement methods cannot provide detailed information on sur- 
face texture or its spatial distribution (e. g. roughness variation) associated with product quality.

\section{Emerging profilometry technologies for PE-coated paperboard characterization}

A recent study compares a set of optical profilometers used to measure the surface roughness of various paper grades (Mettänen and Hirn 2015). It demonstrated that the preferred and selected instrument depends on the spatial distribution of the surface roughness, and systematic deviations were present in all-optical measurement techniques at different stages of the measurements. The surface material's optical and geometrical properties present a challenge when it comes to establishing a common technique for characterizing all paper grades. Optical devices such as confocal scanning microscopes (Jordan et al. 1998) and scanning electron microscopes (SEMs) (Hawkes and Reimer 2013) are valuable tools for surface characterization. Chromatic confocal microscope (CCM) is a variation within the former category of microscopes, and it has been included in the new ISO 25178 Geometric Product Specifications (Blateyron 2011) for areal surface characterization. Up to $1 \mathrm{~cm}^{2}$ (i. e., relatively large) areas of paperboard can be analyzed by scanning the sample's area laterally (i. e., along the Cartesian coordinate axis $X-Y$ ) with micrometer lateral accuracy and a depth resolution of tens of nanometers (Mettänen and Hirn 2015). The high dynamic range of the SEM represents an advantage over other imaging techniques. SEM is employed for the surface characterization of paper and paperboard, with the paper and paperboard sample's cross-section commonly being analyzed under this microscope. This technique provides information about the local structures in the material composition and their relationships with different surface parameters affecting the product quality. Cross-sectional micrographs of paperboard combined with digital image analysis were used to find correlations between the base sheet distribution of the board and its coating thickness uniformity (Dahlström and Uesaka 2009). This approach has also been used to quantify different surface statistical parameters, including surface roughness, of commercial supercalender paper (Chinga et al. 2007). Although these methods provide agreement as to how the local composition affects the paper quality, cross-sectional analysis is timeconsuming and invasive. The sample's preparation can result in a modification of the original structural properties, and the cross-section inspected is limited to small areas of the product.
As an imaging system, an SEM provides a greater dynamic range than does any optical system. Electrons have a shorter wavelength than do photons, allowing the SEM to analyze specimens using a larger field of view with a greater depth of field and greater magnification. It also permits observation of surface details, such as topographic texture and material contrast, up to nanometer resolution. The highly detailed topographic images acquired by an SEM are two-dimensional, and the gray-scale information lacks quantified depth information about the specimen. Software developers are now integrating stereo-photogrammetry to estimate surface topography (Marinello et al. 2008) from SEM micrographs. Several photogrammetry techniques have been proposed, with stereo-photogrammetry being one of the most popular (Howell 1978). Stereo-photogrammetry with an SEM uses two (i. e., a stereo pair) or more images of the same scene acquired from different angles of incidence. In the SEM, a sample mounted on a stage can be oriented towards the detector at different angles, contributing to changes in perspective relative to the same region of interest (ROI). This profilometry technique for characterizing paper samples using the SEM was reported by Enomae (Enomae et al. 1993). It used stereo-photogrammetry after acquiring two images from two detectors positioned at both sides of the electron beam column, generating the disparity map necessary for calculating depth in the sample image. Most recently, instrument inter-comparison using optical coherence scanning interferometry and 3D SEM stereo-photogrammetry for dental implants was presented (Glon et al. 2014). Surface roughness analysis was compared among the instruments, with the author selecting the root mean square roughness (Rq) and power spectral density function (PSD) surface parameters for statistical validation. PSD analysis provided a resource for instrument inter-comparison, using the spatial bandwidth limits of the instruments and the resulting integration limits for surface roughness calculations and to highlight the differences in the results.

Duparré (Duparré etal. 2002) proposed studying larger-scale PSD in the topography of paper samples of various grades. Two profilometers with overlapping spatial bandwidth distributions were employed to extend the spatial wavelength analysis of the PSD. Similarly, multi-scale analysis using a focus variation optical profilometer has been proposed (Vernhes et al. 2008) for the analysis of different grades of papers based on the new ISO 25178 specifications, with each measurement providing scale-limited surfaces within a finite range of spatial distributions. The surface parameters measured within different bandwidth limits describe the instrument performance along with 


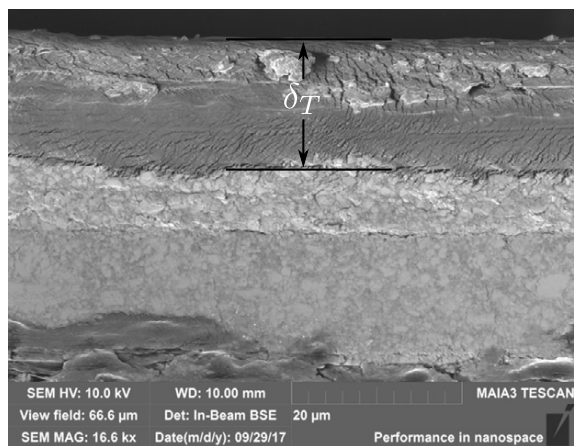

(a)

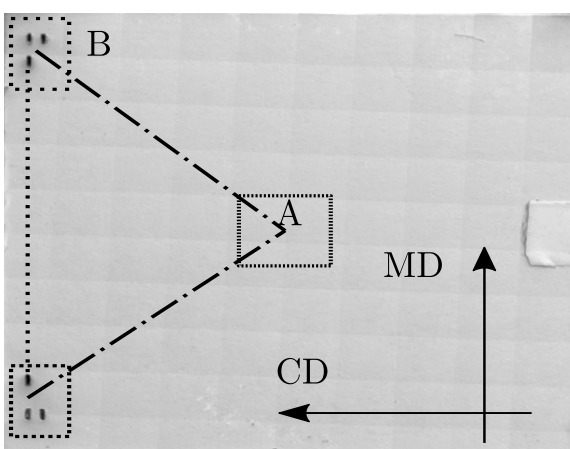

(b)

Figure 1: (a) SEM cross-sectional image showing the material distribution in the paperboard sample. $\delta_{T}$ indicates the PE coating layer with a thickness range of 13 to $15 \mu \mathrm{m}$. (b) The top view of the $20 \times 20 \mathrm{~mm}$ PE-coated paperboard sample. The indentation marks help to identify the center of the sample and its orientation relative to the manufacturing process, i. e., MD and CD.

the different bandwidth limits. An essential part of the inter-instrument comparison is to use the bandwidth limits of the instruments as information for validating the surface parameter characterization. To this end, bandwidth matching guidelines for inter-instrument comparison have been formally introduced by Leach (Leach and Haitjema 2010).

In this study, we compare surface parameters from different scanning imaging systems. The imaging systems project a beam spot on the sample, and the beam size defines both the spatial resolution of the instrument and the spatial bandwidth limits of the measurement. To carry out comparative instrumental studies, matching the number of pixels on the area of comparison is necessary. The spatial resolutions of CCMs and SEMs are limited by this beam spot characteristic and by the spectral wavelength of the particle that generates the image, i.e., photons or electrons.

\section{Outline of the article}

In this study, we measured the surface topography of an industrially manufactured, high-quality PE-coated paperboard using two complementary imaging techniques. The aim was to provide an alternative instrumental technique for the surface characterization of paperboard using 3D stereo-photogrammetry with a scanning electron microscope (SEM) and a valid comparison with reference optical profilometry using a chromatic confocal microscope (CCM). We described the use of the software tool MeX 6.0 (Alicona Imaging $\mathrm{GmbH}$, Raaba/Graz, Austria), as it was included in the SEM software tools for obtaining the surface topography dataset of the sample. After obtaining the surface topography datasets, we calculated the areal surface roughness parameter, Sq, and the 1D-PSD function from both the sample's machine direction (MD) and crossmachine direction (CD) (Alam et al. 2011), using the results for the inter-instrument comparison validation. From the PSD curves, the one-dimensional roughness parameter, Rq, was calculated from both datasets. The following section describes the sample preparation, the instruments used in the measurements, and the methods for surface parameter extraction. Following that, we introduce the topography maps and the results of the metrology analysis. Finally, conclusions and future work are presented.

\section{Materials and methods}

This section presents the sample preparation and instrument selection and description. Furthermore, it describes the statistical parameters used for the analysis and comparison of the topographic datasets.

\section{Samples}

The sample selected for this study is a $20 \times 20 \mathrm{~mm}$ sample from an industrially manufactured low-density polyethylene (PE)-coated paperboard sheet. Figure 1(a) shows the composition of the paperboard sample in a cross-sectional image acquired by an SEM. The thickness, $\delta_{T}$, of the PE coating layer, ranges from $13 \mu \mathrm{m}$ to $15 \mu \mathrm{m}$, corresponding to the expected thickness after the manufacturing process. The surface topography of the sample was measured using two microscopes, i. e., a CCM and an SEM. Figure 1(b) shows the sample being imaged by the SEM under normal 
incidence. We located a region of interest (ROI) (A), used to generate identical topography datasets. To accurately determine the center of the sample where the ROI also has its center, a pattern (B) was engraved using a CNC laser machine to more accurately and precisely locate the marks. The pattern also provides information regarding the orientation of the sample during the manufacturing process, i. e., the machine direction (MD) and cross-machine direction (CD). These directions are important when measuring the statistical parameters on anisotropic surfaces like those found in paper and paperboard. The orientation of the fibers is related to the gloss uniformity and the surface macro-roughness of the paperboard products.

It was necessary to apply a metal coating on the sample surface to obtain high-contrast SEM images while reducing the noise generated by electron scattering from non-conductive materials in the sample. A $3 \mathrm{~nm}$ layer of iridium was sputtered on top of the sample surface; this coating material was selected because its smaller grain size would generate a more even coating distribution, in contrast to other conventional materials such as gold. All measurements used the same PE paperboard sample coated with a top layer of iridium to finally obtain the same surface topography.

\section{Instruments}

\section{Chromatic confocal microscope}

Chromatic confocal microscope (FRT MicroProf; Fries Research \& Technology GmbH, Bergisch Gladbach, Germany) was employed as the reference device with which to obtain the surface texture and surface statistical parameters from the PE-coated paperboard sample; these parameters are included in the new ISO 25178 specifications for both instrument and statistical validation. The CCM is located in a laboratory with controlled and stable temperature and humidity, to ensure the repeatability of the results during the measurement acquisition time. Figure 2 shows a schematic of the CCM. A white light source (400$700 \mathrm{~nm}$ spectral range) is collimated through the system and focused on the inspected surface by a positive chromatic aberration lens. The chromatic aberration in the lens is specifically designed to be as great as possible, dispersing the light spectral range along with the beam spot. The light is reflected into the sensor, and an intensity peak centered at a particular wavelength in the system's spectral range represents the local height variation of the sample. Light travels through a pinhole detector placed in front of a spectrometer. The spectrometer decodes the spectral in-

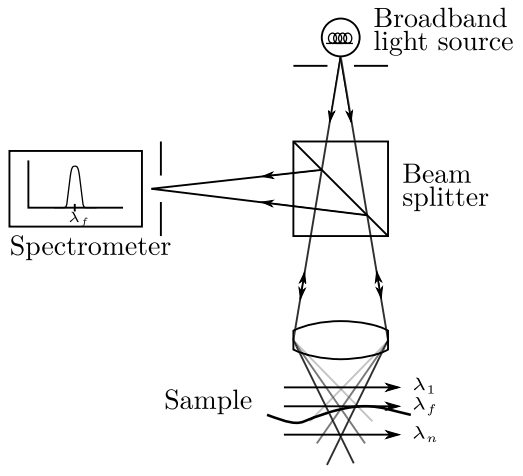

Figure 2: Chromatic confocal microscope.

formation as the local height variation from the calibrated spectral range. The optical microscope employs no moving parts to obtain the local height, but it does require that the base move the sample in the $X-Y$ direction until the entire area is scanned. A chromatic probe with a calibrated depth range of $300 \mu \mathrm{m}$ and lateral resolution (i. e., beam spot size) of $1 \mu \mathrm{m}$ was used as the objective sensor in the CCM.

The grating spectrometer provides instantaneous depth information, making such devices much faster than traditional confocal microscopes or profilometer techniques. The range of heights that this instrument can measure is limited by the design of the dispersive objective and the spectral range of the light source. Another drawback of this system is its limited ability to measure topographies in objects with steep local variations.

\section{Scanning electron microscope}

Two-dimensional SEM micrographs of the sample were acquired using a TESCAN MIRA3 GMU (TESCAN Brno, s. r. o., Brno, Czechia). Multiple detectors are available for this SEM, and a secondary electron detector was chosen for pure topographic imaging. The electron beam voltage of $2 \mathrm{kV}$ was selected, which corresponds to a beam spot size of $25 \mathrm{~nm}$. To create the stereo-pair images, the eucentric tilting of the sample stage was used with a tilt of $\pm 5^{\circ}$ from a central tilted position of $20^{\circ}$. The sample stage could be tilted towards the detector to increase the electron yield from the sample-detector interaction and to obtain higher topographic contrast in the images. Before scanning the surface, it is necessary to co-localize the ROI. We used the MeX software co-localization tool, placing a 2D image from the CCM-measured topography into the live image from the SEM. The central tilted position (i. e., at $20^{\circ}$ ) described above was aligned with the texture information from the CCM image in the software. The texture information pro- 


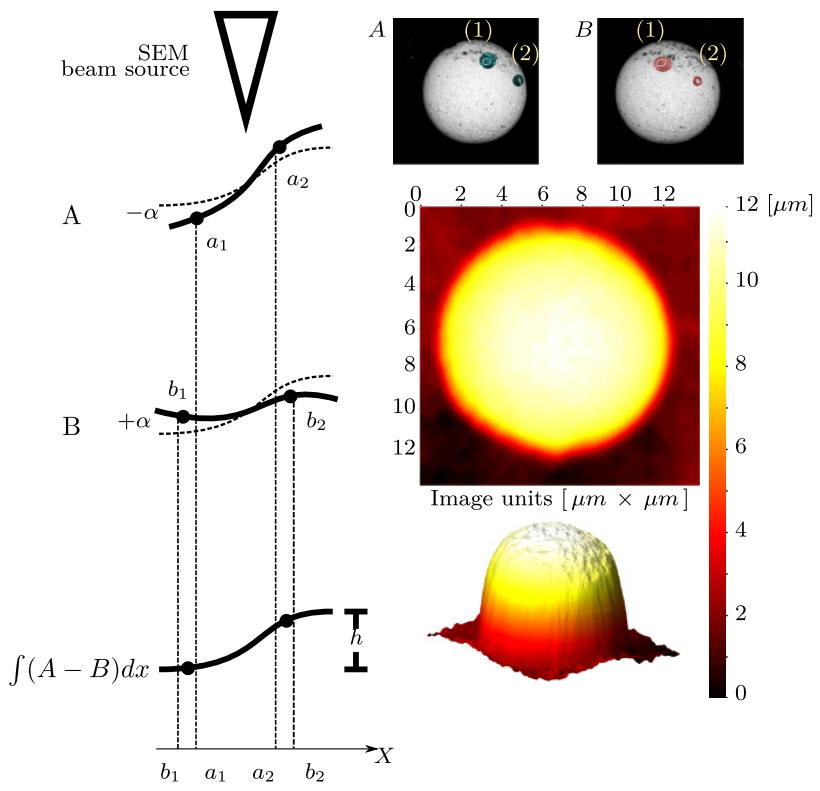

Figure 3: SEM stereo-photogrammetry requires at least two images from different perspectives, i. e., $A$ and $B$. Image pixels in both images undergo lateral displacement, i. e., (1) and (2). By knowing how far identified features are displaced and the angle of perspective, $\pm \alpha$, triangulation can be used to calculate the height $(h)$ in every pixel.

vided the necessary fiducial marks to center the SEM micrographs for the CCM measurement. The obtained stereopair SEM micrographs used a magnification of $554 \times$ with a field of view of $2 \mathrm{~mm}$. The two resulting micrographs were cropped to a $4096 \times 4096$ pixel area with $488.28 \mathrm{~nm}$ lateral resolution.

\section{SEM stereo-photogrammetry}

After locating ROI on the SEM live image and aligning with the CCM 2D image, stereo-pair images were acquired from two perspectives $\pm 5^{\circ}$ from a central tilted position of $20^{\circ}$. As shown in Figure 3, the stereo-pair images were used to create a disparity map showing the lateral distances of identical features located at slightly different positions in the two images. In Figure 3, a reference standard sphere consisting of Polystyrene Divinylbenzene and traceable diameter of $10 \mu \mathrm{m}$ (4D-10, 4D Series Dry Microsphere Size Standards NIST, Thermo Scientific ${ }^{\mathrm{TM}}$ ) is captured on the SEM and arranged with relatively different orientations in each image. To estimate the 3D topography, the disparity map with the known tilt angles was used to calculate the topographic maps by triangulation. The sphere can be reconstructed in its 3D shape, but not the areas below the sphere occluded to the sensor. From the SEMs toolbox, we used the software MeX 6.0 (Alicona Imaging $\mathrm{GmbH}$, Raaba/Graz, Austria) as a standard to create the topographic datasets. We downscaled the dataset using bilinear spline interpolation after the topographic maps were created. This step was necessary to adjust the lateral resolution of the CCMs topographic dataset.

\section{The statistical parameters}

To extract the different spatial components on a topographic dataset, different spatial filters are used. In the context of areal surface characterization, two filters are used, the S-filter and the L-filter. The former removes small-scale lateral components such as residual noise, and the latter limits the effects of large-scale components in the surface texture. The application of these filters results in a scale-limited surface. To obtain scale-limited surfaces from the topographic datasets, the general procedure presented in the ISO 25178-3 specifications (ISO 25178-3 2012) was followed. Second-order polynomial regression was used to derive the form from the extracted surfaces. The use of $S$ and $L$ Gaussian filters with $2.5 \mu \mathrm{m}$ and $250 \mu \mathrm{m}$ nesting indexes, respectively, resulted in a scale-limited (S-L) surface dataset. The lateral resolution of the CCM dataset, i. e., $1.36 \mu \mathrm{m}$, was used as the minimum possible value for the S-filter nesting index. Importantly for the bandwidth matching condition, each extracted dataset used for this comparison had the same number of pixels and equal lateral resolution. The generated S-L surface was conformable with the bandwidth characteristics of both profilometry systems.

Areal parameters, introduced in ISO 25178-2, are used for analysing surface texture. The areal root mean square surface heights (Sq) and the 1D power spectral density (1DPSD) function in the axial directions, i. e., $C D$ and MD, were calculated from the obtained S-L surface. The Sq parameter served as a comparison tool for analysing the overall areal roughness. However, it does not take into consideration directional features such as those encountered on anisotropic surfaces. To account for the directional anisotropy of the samples, the 1D-PSD 1 is used within the spatial bandwidth limits of the instruments, and the integration of the curve in the 1D-PSD results in the RMS surface roughness (2) for the MD and the $\mathrm{CD}$.

$$
\begin{aligned}
& P S D\left(f_{x}\right)=\frac{\delta_{x}}{N}|F F T|^{2} \\
& \sigma_{r m s}^{2}=\int_{f_{\min }}^{f_{\text {max }}} P S D(f) d f
\end{aligned}
$$



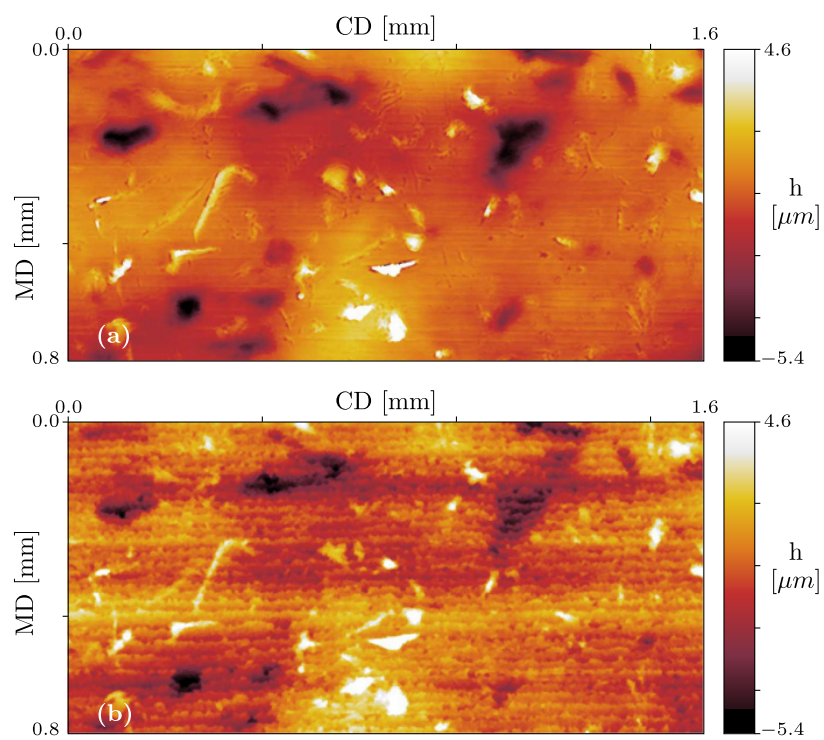

Figure 4: The primary surface resulting from the sample topography measurement using (a) a CCM and (b) a 3D stereo-photogrammetry SEM. An S-filter with a $2.5 \mu \mathrm{m}$ nesting index was used in the primary and, later, in a second-order polynomial regression to extract the form from the filtered dataset.

where $\delta_{x}$ is the lateral resolution of the system, $N$ the number of pixels in the measurement direction, and $|F F T|^{2}$ the fast Fourier transform of the S-L surface. The result of integrating the PSD is the profile parameter, $\sigma_{r m s}$, analogous to the one-dimensional rms roughness, $\mathrm{Rq}$.

\section{Results and discussion}

\section{Topography dataset generation and representation}

The surface topography of one PE-coated paperboard sample was determined using CCM and 3D SEM stereophotogrammetry measurement techniques. The results are comparable only if the instruments' spatial wavelengths match within the bandwidth limits of the measurements, so special care is taken to use appropriate filtering techniques to ensure adequate resulting topographies, as described in the "Materials and methods" section.

Figure 4 presents the surface topography of the sample. The region of interest (ROI) has been cropped to 1.6 $\times 0.8 \mathrm{~mm}$. First, a low-pass filter (S-filter) with a $2.5 \mu \mathrm{m}$ nested index was used and next a second-order polynomial regression to remove the form in the surface was applied to the topographic datasets to obtain the presented primary surfaces.

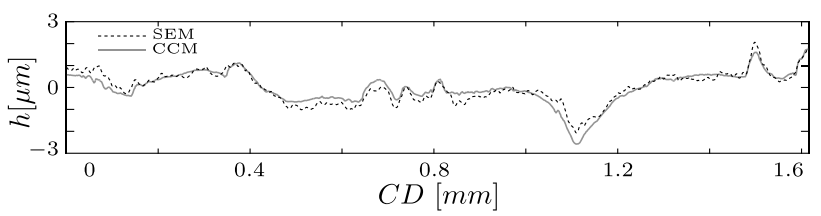

Figure 5: A total of 128 averaged profiles from the S-F surface. The profiles were selected across the $C D(\mathrm{~mm})$ with topographic average heights $\mathrm{h}[\mu \mathrm{m}]$.

Close agreement was found between the topographic datasets obtained from measurements using the two profilometry techniques. While the CCM benefits from the smooth surface of the PE-coated paperboard, allowing accurate measurement of the sample topography, SEM stereo-photogrammetry does not. The image shows that the topography obtained using the SEM stereophotogrammetry technique suffers from uses artefacts. These artefacts formed on the axis along which the sample stage was tilted during stereo-pair image acquisition. Figure 4(b) is aligned with the $\mathrm{CD}$ of the paperboard manufacturing process. The many flat areas encountered on the surface and the low magnification of the SEM images affect the parallax photogrammetry conditions when estimating the disparity map, amplifying the artefact propagation along with the topography. This is a known limitation of photogrammetry techniques (Pouchou et al. 2002).

A long-pass filter (L-Filter) with a $250 \mu$ m nesting index was applied to the primary surfaces obtained from both datasets, resulting in the bandwidth-matched S-L surface necessary for the statistical validation used during instrument inter-comparison. In total, 128 one-dimensional height profiles along the $\mathrm{CD}$ (i. e., horizontal axis) were extracted and averaged. Figure 5 compares the averaged profiles obtained from both measurement techniques. From the curves, in Figure 5, when calculated the normalized root mean square surface roughness of the averaged profiles, it shows that obtained surface roughness from the SEM topography is $6 \%$ greater than that obtained using the reference method.

Finally, Sq was calculated as well as the 1D-PSD in both machine directions on both the S-L surfaces, as shown in Figure 6. From the resulting 1D-PSD, we calculated the surface roughness parameter, $\sigma_{r m s}$, in each direction on the surface. Table 1 presents the resulting areal parameter, Sq, and the roughness value obtained after integrating the 1D-PSD curves from Equation (2), in both MD and $\mathrm{CD}$.

The roughness values from the surface texture, Sq, and the one-dimensional surface roughness, $\sigma_{r m s}$ in 


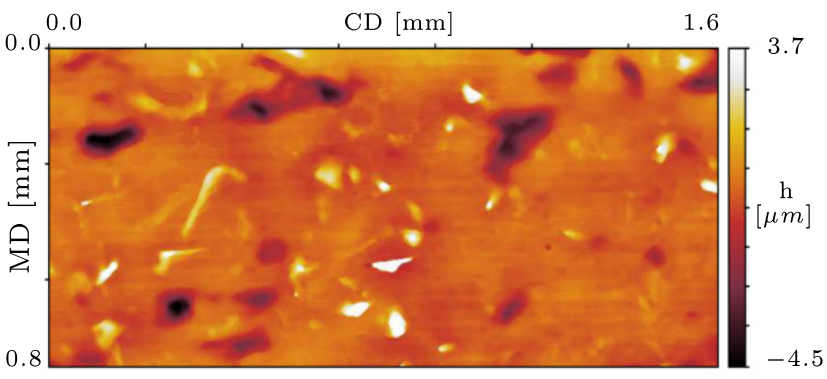

(a) $\mathrm{CCM}$

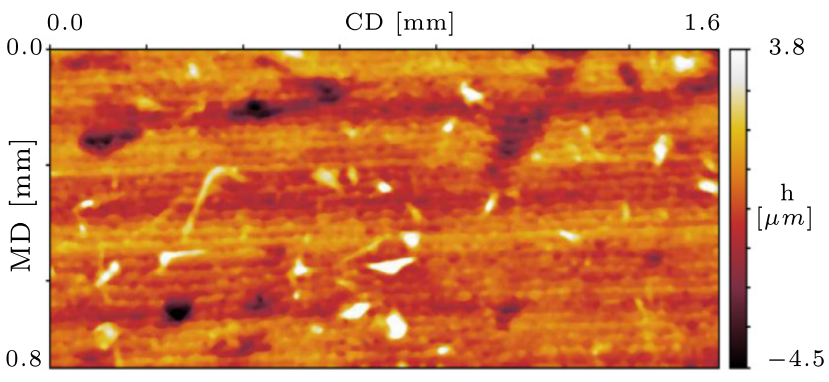

(b) SEM

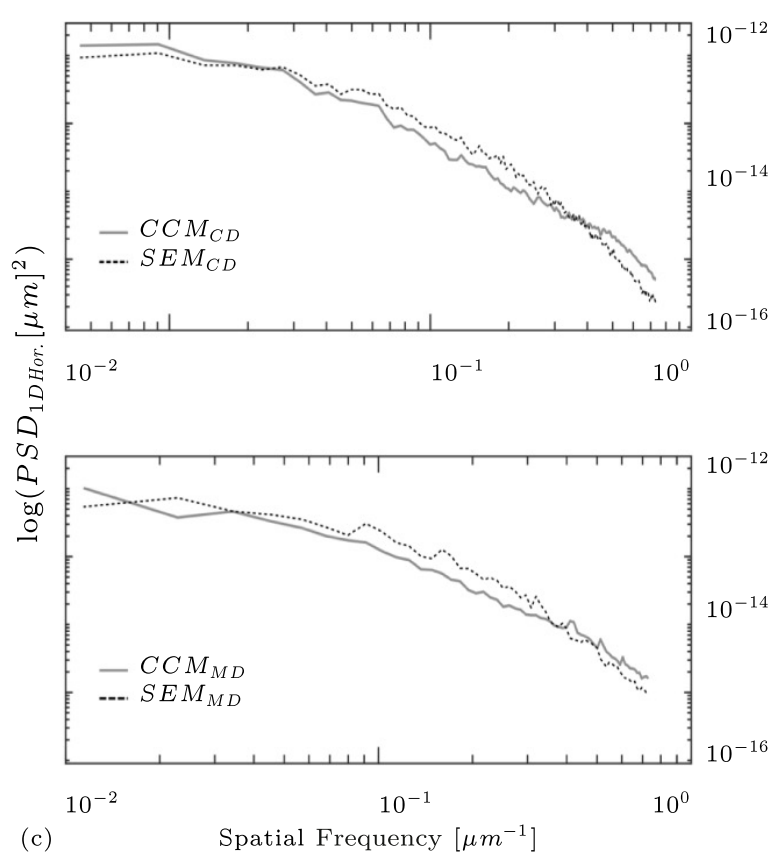

(c)

Figure 6: Roughness component of S-L surface obtained from (a) CCM and (b) SEM stereo-photogrammetry topography datasets. PSD waS calculated from both topographies (c) in CD and MD to directly compare the orthogonal texture behaviour.

Table 1: Calculated statistical parameters: Sq from areal topography and $\sigma_{r m s}$ from the 1D-PSD in MD and CD.

\begin{tabular}{rrrr}
\hline & Sq $[\mu \mathrm{m}]$ & $\sigma_{C D}[\mu \mathrm{m}]$ & $\sigma_{M D}[\mu \mathrm{m}]$ \\
\hline SEM & 1.07 & 1.05 & 1.14 \\
CCM & 0.97 & 1.11 & 0.94 \\
\hline
\end{tabular}

the MD, obtained using the SEM stereo-photogrammetry dataset, are higher than those obtained using the reference method, while $\sigma_{r m s}$ in the CD from the same dataset is smaller. The difference in the surface roughness calculated from the averaged profiles is larger in the SEM than in the CCM datasets but is measured from a small area. By using the 1D-PSD, we can calculate the surface roughness of the complete dataset and simultaneously separate the effects from the anisotropic behavior of the samples by separately measuring the 1D-PSD in both CD and MD (Duparré et al. 2002).

We conclude that the calculated statistical parameters in both topographic datasets generally agree well. The differences found when comparing the areal parameter Sq and $\sigma_{r m s}$ in MD are related to the artefacts and noise resulting from the SEM topography dataset. According to our observations, this is related to the direction of the stereo pair projection in the stereo-photogrammetry technique SEM.

\section{Conclusion}

SEM stereo-photogrammetry has been proposed as a profilometry technique for the surface roughness characterization of high-quality PE-coated paperboard. We demonstrated that this imaging tool in combination with the procedure to produce topographic height information can be used as a profilometry instrument in the paper and paperboard industry. In our study, surface roughness measurements from a reference profilometry method, i. e., CCM, were compared with those from the SEM stereophotogrammetry topography of PE-coated paperboard, with special attention paid to implementing a bandwidth matching strategy, essential for valid instrument intercomparison. Our results indicate good agreement between both topography datasets, and validation from the reference method indicates that SEM stereo-photogrammetry can be used for the surface roughness characterization of PE-coated paperboard. Despite the small differences on the surface parameter resulted from the artefacts inherent to the photogrammetry technique, affecting the accuracy of the results, the technique's short acquisition time versus that of the reference method can be a key point in selecting one method over the other. Our study opens up the possibility of investigating the effects of different SEM parameters on the accuracy of the profilometry results. In a fu- 
ture study, we propose comparing different settings based on the SEM's magnification, tilting angle, and voltage of the beam source, which could lead to improvements in the accuracy of the stereo-photogrammetry technique and compared a larger set of produced paperboard samples, including laminated paperboard composed of pigmented coatings.

Funding: This research received no external funding.

Conflict of interest: The authors declare no conflict of interest.

\section{References}

Alam, A., Thim, J., Manuilskiy, A., O’Nils, M., Westerlind, C., Lindgren, J., Liden, J. (2011) Investigation of the surface topographical differences between the Cross Direction and the Machine Direction for newspaper and paperboard. Nord. Pulp Pap. Res. J. 26(4):468-475.

Béland, M.-C., Bennett, J.M. (2000) Effect of local microroughness on the gloss uniformity of printed paper surfaces. Appl. Opt. 39(16):2719.

Blateyron, F. (2011) Chromatic Confocal Microscopy. In: Optical Measurement of Surface Topography. Springer Berlin Heidelberg, Berlin, Heidelberg. pp. 71-106.

Chinga, G., Johnsen, P.O., Dougherty, R., Berli, E.L., Walter, J. (2007) Quantification of the 3D microstructure of SC surfaces. J. Microsc. 227(3):254-265.

Dahlström, C., Uesaka, T. (2009) New insights into coating uniformity and base sheet structures. Ind. Eng. Chem. Res. 48(23):10472-10478.

Duparré, A., Ferre-Borrull, J., Gliech, S., Notni, G., Steinert, J., Bennett, J.M. (2002) Surface characterization techniques for determining the root-mean-square roughness and power spectral densities of optical components. Appl. Opt. 41(1):154.

Enomae, T., Onabe, F. (1997) Characteristics of Parker Print-Surf roughness as compared with Bekk smoothness. J. Fiber Sci. Technol. 53(3):86-95.

Enomae, T., Onabe, F., Usuda, M. (1993) Application of new profilometry using topographic scanning electron microscope to paper surface topography. Tappi J. 76(1):85-90.

Glon, F., Flys, O., Lööf, P.J., Rosén, B.G. (2014) 3D SEM for surface topography quantification - A case study on dental surfaces. J. Phys. Conf. Ser. 483(1):012026.

Hawkes, P.W., Reimer, L. Scanning Electron Microscopy: Physics of Image Formation and Microanalysis. Springer Series in Optical Sciences. Springer Berlin Heidelberg, 2013.

Howell, P.G. (1978) The derivation of working formulae for SEM photogrammetry at low instrumental magnifications. Scanning 1(4):230-232.

ISO 25178-3 (2012) Geometrical product specifications (GPS) - Surface texture: Areal Part 3: Specification operators. International Standards Organization, Geneva, Switzerland. Jordan, H.J., Wegner, M., Tiziani, H. (1998) Highly accurate non-contact characterization of engineering surfaces using confocal microscopy. Meas. Sci. Technol. 9(7):1142-1151.

Leach, R., Haitjema, H. (2010) Bandwidth characteristics and comparisons of surface texture measuring instruments. Meas. Sci. Technol. 21(3):032001.

Marinello, F., Bariani, P., Savio, E., Horsewell, A., De Chiffre, L. (2008) Critical factors in SEM 3D stereo microscopy. Meas. Sci. Technol. 19(6):065705.

Mettänen, M., Hirn, U. (2015) A comparison of five optical surface topography measurement methods. Tappi J. 14(1):27-38.

Pouchou, J.L., Boivin, D., Beauchêne, P., Le Besnerais, G., Vignon, F. (2002) 3D reconstruction of rough surfaces by SEM stereo imaging. Mikrochim. Acta 139(1-4):135-144.

Vernhes, P., Bloch, J.F., Mercier, C., Blayo, A., Pineaux, B. (2008) Statistical analysis of paper surface microstructure: A multi-scale approach. Appl. Surf. Sci. 254(22):7431-7437. 